\title{
Session E
}

\section{Comparison with observations}


N. E. Piskunov, W. W. Weiss, D. F. Gray, eds.

\title{
Atomic Physics Data for Stellar Atmospheres Research
}

\author{
Charles R. Cowley \\ University of Michigan, Ann Arbor, MI 48109-1090,USA
}

Saul J. Adelman

The Citadel, Charleston, SC 29409-0270, USA

Donald J. Bord

University of Michigan, Dearborn, MI 48128-1491, USA

\begin{abstract}
.
The review will cover the following topics: (1) Ionization energies; (2) Partition functions; (3) Sources of data for atomic and ionic wavelengths, transition probabilities, and broadening parameters, including nuclear effects (hfs and isotope shifts); (4) Opacities from photoionization of abundant elements (atoms and atomic ions) with emphasis on integration of TOPBASE material; and (5) Data bases for diatomic molecules. We emphasize topics of direct relevance to the synthesis of stellar spectra, primarily within the domain where LTE is useful. Additional parameters, such as line-broadening parameters, or excitation cross sections are not reviewed.
\end{abstract}

\section{Ionization Energies}

We provide a table of ionization energies from reliable secondary sources. First and second spectra are from the web pages of NIST (Sansonetti and Martin 2002). Most of the values for the third and fourth spectra are from the Handbook of Chemistry and Physics, 3rd Electronic Edition: (http://www.hbcpnetbase.com/hbcp/), but with a few additions from Dappen(2000) and Cowan (1981, Table 1-1). For the first and second spectra, only a few values differ by more than a few hundredths of an $\mathrm{eV}$ from those given by Moore (1970). Notable changes in the first ionization energies are for $\mathrm{Fe} \mathrm{I}(7.902$ instead of 7.87), YI (6.217 instead of 6.38), and Zr I (6.634 instead of 6.84), all values in $\mathrm{eV}$. 
Table 1. Ionization energies for the first through fifth spectra

\begin{tabular}{|c|c|c|c|c|c|c|}
\hline Element & $\mathrm{Z}$ & $I$ & $I I$ & $I I I$ & $I V$ & $V$ \\
\hline$\overline{\text { Hydrogen } \mathrm{H}}$ & 1 & 13.5984 & & & & \\
\hline Helium $\mathrm{He}$ & 2 & 24.5874 & 54.417760 & & & \\
\hline Lithium Li & 3 & 5.3917 & 75.6400 & 122.45429 & & \\
\hline Beryllium Be & 4 & 9.3227 & 18.21114 & 153.89661 & 217.71865 & \\
\hline Boron B & 5 & 8.2980 & 25.1548 & 37.93064 & 259.37521 & 340.22580 \\
\hline Carbon $\mathrm{C}$ & 6 & 11.2603 & 24.3833 & 47.8878 & 64.4939 & 392.087 \\
\hline Nitrogen $\mathrm{N}$ & 7 & 14.5341 & 29.6013 & 47.44924 & 77.4735 & 97.8902 \\
\hline Oxygen $\mathrm{O}$ & 8 & 13.6181 & 35.1211 & 54.9355 & 77.41353 & 113.8990 \\
\hline Fluorine $\mathrm{F}$ & 9 & 17.4228 & 34.9708 & 62.7084 & 87.1398 & 114.2428 \\
\hline Neon $\mathrm{Ne}$ & 10 & 21.5646 & 40.96296 & 63.45 & 97.12 & 126.21 \\
\hline Sodium $\mathrm{Na}$ & 11 & 5.1391 & 47.2864 & 71.6200 & 98.91 & 138.40 \\
\hline Magnesium Mg & 12 & 7.6462 & 15.03527 & 80.1437 & 109.2655 & 141.27 \\
\hline Aluminum $\mathrm{Al}$ & 13 & 5.9858 & 18.82855 & 28.44765 & 119.992 & 153.825 \\
\hline Silicon $\mathrm{Si}$ & 14 & 8.1517 & 16.34584 & 33.49302 & 45.14181 & 166.767 \\
\hline Phosphorus P & 15 & 10.4867 & 19.7695 & 30.2027 & 51.4439 & 65.0251 \\
\hline Sulfur S & 16 & 10.3600 & 23.33788 & 34.79 & 47.222 & 72.5945 \\
\hline Chlorine $\mathrm{Cl}$ & 17 & 12.9676 & 23.8136 & 39.61 & 53.4652 & 67.8 \\
\hline Argon Ar & 18 & 15.7596 & 27.62965 & 40.74 & 59.81 & 75.02 \\
\hline Potassium K & 19 & 4.3407 & 31.63 & 45.806 & 60.91 & 82.66 \\
\hline Calcium Ca & 20 & 6.1132 & 11.87172 & 50.9131 & 67.27 & 84.50 \\
\hline Scandium Sc & 21 & 6.5615 & 12.79977 & 24.75666 & 73.4894 & 91.65 \\
\hline Titanium Ti & 22 & 6.8281 & 13.5755 & 27.4917 & 43.2672 & 99.30 \\
\hline Vanadium $\mathrm{V}$ & 23 & 6.7462 & 14.618 & 29.311 & 46.709 & 65.2817 \\
\hline Chromium $\mathrm{Cr}$ & 24 & 6.7665 & 16.4857 & 30.96 & 49.16 & 69.46 \\
\hline Manganese Mn & 25 & 7.4340 & 15.6400 & 33.668 & 51.2 & 72.4 \\
\hline Iron $\mathrm{Fe}$ & 26 & 7.9024 & 16.1877 & 30.652 & 54.8 & 75.0 \\
\hline Cobalt CO & 27 & 7.8810 & 17.084 & 33.50 & 51.3 & 79.5 \\
\hline Nickel Ni & 28 & 7.6398 & 18.16884 & 35.19 & 54.9 & 76.06 \\
\hline Copper $\mathrm{Cu}$ & 29 & 7.7264 & 20.2924 & 36.841 & 57.38 & 79.8 \\
\hline Zinc $\mathrm{Zn}$ & 30 & 9.3942 & 17.96439 & 39.723 & 59.4 & 82.6 \\
\hline Gallium Ga & 31 & 5.9993 & 20.51514 & 30.71 & 64 & 87 \\
\hline Germanium Ge & 32 & 7.8994 & 15.93461 & 34.2241 & 45.7131 & 93.5 \\
\hline Arsenic As & 33 & 9.7886 & 18.5892 & 28.351 & 50.13 & 62.63 \\
\hline Selenium Se & 34 & 9.7524 & 21.19 & 30.8204 & 42.9450 & 68.3 \\
\hline Bromine $\mathrm{Br}$ & 35 & 11.8138 & 21.591 & 36 . & 47.3 & 59.7 \\
\hline Krypton $\mathrm{Kr}$ & 36 & 13.9996 & 24.35984 & 36.950 & 52.5 & 64.7 \\
\hline Rubidium Rb & 37 & 4.1771 & 27.2895 & 40 & 52.6 & 71.0 \\
\hline Strontium Sr & 38 & 5.6949 & 11.0301 & 42.89 & 57 & 71.6 \\
\hline Yttrium Y & 39 & 6.2171 & 12 & 20.52 & 60.597 & 77.0 \\
\hline Zirconium Zr & 40 & 6.6339 & 13.1 & 22.99 & 34.34 & 80.348 \\
\hline Niobium $\mathrm{Nb}$ & 41 & 6.7589 & 14.0 & 25.04 & 38.3 & 50.55 \\
\hline Molybdenum Mo & 42 & 7.0924 & 16.16 & 27.13 & 46.4 & 54.49 \\
\hline Technetium Tc & 43 & 7.28 & 15.26 & 29.54 & 46 & 55 \\
\hline Ruthenium $\mathrm{Ru}$ & 44 & 7.3605 & 16.76 & 28.47 & 50 & 60 \\
\hline Rhodium Rh & 45 & 7.4589 & 18.08 & 31.06 & 48 & 65 \\
\hline Palladium Pd & 46 & 8.3369 & 19.43 & 32.93 & 53 & 62 \\
\hline Silver Ag & 47 & 7.5762 & 21.47746 & 34.83 & 56 & 68 \\
\hline Cadmium Cd & 48 & 8.9938 & 16.90831 & 37.48 & 59 & 72 \\
\hline Indium In & 49 & 5.7864 & 18.8703 & 28.03 & 54.4 & 77 \\
\hline Tin Sn & 50 & 7.3439 & 14.6322 & 30.50260 & 40.73502 & 72.28 \\
\hline
\end{tabular}


Table 1. Ionization energies for the first through fifth spectra (continued)

\begin{tabular}{|c|c|c|c|c|c|c|}
\hline Element & $\mathrm{Z}$ & $I$ & $I I$ & $I I I$ & $I V$ & $V$ \\
\hline Antimony Sb & 51 & 8.6084 & 16.63 & 25.3 & 44.2 & 56 \\
\hline Tellurium Te & 52 & 9.0096 & 18.6 & 27.96 & 37.41 & 58.75 \\
\hline Iodine I & 53 & 10.4513 & 19.1313 & 33 & 42 & 66 \\
\hline Xenon Xe & 54 & 12.1298 & 20.9750 & 32.1230 & 46 & 57 \\
\hline Cesium Cs & 55 & 3.8939 & 23.15744 & 35 & 46 & 62 \\
\hline Barium Ba & 56 & 5.2117 & 10.00383 & 35.84 & 49 & 62 \\
\hline Lanthanum La & 57 & 5.5769 & 11.059 & 19.1773 & 49.95 & 61.6 \\
\hline Cerium Ce & 58 & 5.5387 & 10.85 & 20.198 & 36.758 & 65.55 \\
\hline Praseodymium Pr & 59 & 5.473 & 10.55 & 21.624 & 38.98 & 57.53 \\
\hline Neodymium Nd & 60 & 5.5250 & 10.72 & 22.1 & 40.4 & 60.00 \\
\hline Promethium Pm & 61 & 5.582 & 10.90 & 22.3 & 41.1 & 61.69 \\
\hline Samarium Sm & 62 & 5.6436 & 11.07 & 23.4 & 41.4 & 62.66 \\
\hline Europium Eu & 63 & 5.6704 & 11.25 & 24.92 & 42.7 & 63.23 \\
\hline Gadolinium Gd & 64 & 6.1501 & 12.09 & 20.63 & 44.0 & 64.76 \\
\hline Terbium Tb & 65 & 5.8638 & 11.52 & 21.91 & 39.79 & 66.46 \\
\hline Dysprosium Dy & 66 & 5.9389 & 11.67 & 22.8 & 41.47 & 62.08 \\
\hline Holmium Ho & 67 & 6.0215 & 11.80 & 22.84 & 42.5 & 63.93 \\
\hline Erbium Er & 68 & 6.1077 & 11.93 & 22.74 & 42.7 & 65.10 \\
\hline Thulium Tm & 69 & 6.1843 & 12.05 & 23.68 & 42.7 & 65.42 \\
\hline Ytterbium Yb & 70 & 6.2542 & 12.176 & 25.05 & 43.56 & 65.58 \\
\hline Lutetium Lu & 71 & 5.4259 & 13.9 & 20.9594 & 45.25 & 66.8 \\
\hline Hafnium Hf & 72 & 6.8251 & 15 & 23.3 & 33.33 & 68.38 \\
\hline Tantalum Ta & 73 & 7.5496 & 16 & 22 & 33 & 45 \\
\hline Tungsten W & 74 & 7.8640 & 16.1 & 24 & 35 & 48 \\
\hline Rhenium Re & 75 & 7.8335 & 17 & 26 & 38 & 51 \\
\hline Osmium Os & 76 & 8.4382 & 17 & 25 & 40 & 54 \\
\hline Iridium Ir & 77 & 8.9670 & 17 & 27 & 39 & 57 \\
\hline Platinum Pt & 78 & 8.9587 & 18.563 & 28 & 41 & 55 \\
\hline Gold Au & 79 & 9.2255 & 20.20 & 30 & 44 & 58 \\
\hline Mercury $\mathrm{Hg}$ & 80 & 10.4375 & 18.7568 & 34.2 & 46 & 61 \\
\hline Thallium $\mathrm{Tl}$ & 81 & 6.1082 & 20.4283 & 29.83 & 50.7 & 64 \\
\hline Lead $\mathrm{Pb}$ & 82 & 7.4167 & 15.03248 & 31.9373 & 42.32 & 68.8 \\
\hline Bismuth $\mathrm{Bi}$ & 83 & 7.2856 & 16.703 & 25.56 & 45.3 & 56.0 \\
\hline Polonium Po & 84 & $8.417 ?$ & 19 & 27 & 38 & 61 \\
\hline Astatine At & 85 & 9.3 & 20 & 29 & 41 & 51 \\
\hline Radon Rn & 86 & 10.7485 & 21 & 29 & 44 & 55 \\
\hline Francium Fr & 87 & 4.0727 & 22 & 33 & 43 & 59 \\
\hline Radium Ra & 88 & 5.2784 & 10.14715 & 34 & 46 & 58 \\
\hline Actinium Ac & 89 & 5.17 & 11.75 & 20 & 49 & 62 \\
\hline Thorium Th & 90 & 6.3067 & 11.9 & 20.0 & 28.8 & 65 \\
\hline Protactinium & 91 & 5.89 & & & & \\
\hline Uranium U & 92 & 6.1941 & 10.6 & & & \\
\hline Neptunium Np & 93 & 6.2657 & & & & \\
\hline Plutonium $\mathrm{Pu}$ & 94 & 6.0262 & 11.2 & & & \\
\hline Americium Am & 95 & 5.9738 & & & & \\
\hline Curium Cm & 96 & 5.9915 & & & & \\
\hline Berkelium Bk & 97 & 6.1979 & & & & \\
\hline Californium Cf & 98 & 6.2817 & 11.8 & & & \\
\hline Einsteinium Es & 99 & 6.42 & 12.0 & & & \\
\hline Fermium Fm & 100 & 6.50 & & & & \\
\hline
\end{tabular}




\section{Partition Functions}

\subsection{General Remarks}

Abundance codes currently in use employ a variety of algorithms to calculate partition functions. Some of the routines are decades old. The general method of calculating a sum over the known states has been known since the early days of quantum mechanics. However, our knowledge of the locations of these states and the computational power to take them into account has changed significantly. Abundance workers would do well to make sure they are using the most complete and accurate material, as well as modern techniques. References to some of the older work on partition functions are given by Gray (1992) and Cowley and Adelman (1983). Other algorithms recently used are those of Traving, Baschek, and Holweger (1966), or Irwin (1981).

\subsection{Influence of Completeness on Partition Functions}

The degree to which the level structure of a given ion is complete, particularly below about $30000 \mathrm{~cm}^{-1}$, is critical to the reliability of the partition function for the species. For example, Bord and Cowley (2002) have shown that, at the temperature of the Sun, the partition function for Ho II increases by a factor of nearly 1.5 over that inferred from the 49 published levels (Martin, Zalubas, and Hagan 1978) when contributions from unobserved levels arising from the $\left(4 f^{11} 6 p+4 f^{10}(5 d+6 s)^{2}\right)$ group are included. The impact of such changes for stellar abundance work can be quite significant because the number of absorbers scales inversely with the partition function. In the case of holmium in the Sun, raising the partition function by $50 \%$ requires an increase in the abundance of this element of 0.17 dex in order to match the strengths of observed lines; this alone is enough to nearly reconcile the photospheric abundance of holmium based on earlier work (Deams, Biémont, and Grevesse 1984) with that found in CI meteorites.

Cowan (1981, Table 1-4) has assessed the analyses of atomic spectra in his Table 1-4, using a letter scale in which A signifies an essentially complete analysis and $\mathrm{F}$ identifies cases where no individual lines are known, only unresolved transition arrays. The Ho II spectrum, for example, is graded C, with only a few dozen or so levels known. Several of the third spectra of the lanthanides were graded C or "worse" by Cowan, leading to the study by Cowley and Barisciano (1994). They used Cowan's code to complete the level structure of the third spectra of La through Lu for temperatures up to $31,000 \mathrm{~K}$. This led to significant revisions of a number of the calculated partition functions at $10,000 \mathrm{~K}$ (and above). They gave Bolton (1970) coefficients, but the energy levels are available from CRC.

We have recently investigated the completeness of the first and second spectra of the 5d-elements tungsten, rhenium, and osmium for the purpose of reevaluating the partition functions for these ions. The spectra of all of these species are rated as B (all of the lowest 3 or 4 configurations and portions of some higher ones are known) by Cowan, except for Os II which is graded C.

As features attributable to these ions are increasingly being identified in high resolution, near-ultraviolet spectra of warm stars and used in abundance work (cf. Cowan et al. 2002, Henderson et. al. 1999, Leckrone et al. 1999, and 
Wahlgren et al. 1997, 1998), it is worthwhile to re-visit this issue. Inaccuracies in the partition function systematically affect abundances derived from all spectral lines. Thus, as for the ionization potentials, which, for the 5d-elements, are also undergoing revision (see, for example, Campbell-Miller and Simard 1996), care must be taken to use the best available partition functions. In particular, we demonstrate that relying on partition functions based only on the data included in Atomic Energy Levels, Volume III (Moore 1958, hereafter AEL), can lead to errors of $\approx 10 \%$ or more in some cases for temperatures of $10000 \mathrm{~K}$ and higher. In the following paragraphs, we briefly summarize the status of our investigations.

W I: AEL gives a total of 354 levels, 61 of which are below $30000 \mathrm{~cm}^{-1}$. Most of the low-lying levels belong to configurations of even parity. Laun and Corliss (1968) re-analyzed the spectrum adding 37 new even levels and 64 new odd levels. Shadmi and Caspi (1969) published a theoretical investigation of the low-lying even levels arising from the $(5 \mathrm{~d}+6 \mathrm{~s})^{6}$ group which provided an interpretation for every level found experimentally below $40000 \mathrm{~cm}^{-1}$ and predicted the existence of numerous other levels. Based on these predictions, Corliss (1969) was able to identify ten new levels in W I.

We have augmented the AEL data by including all the experimental and theoretical levels below $40000 \mathrm{~cm}^{-1}$ identified in the above references, and have recomputed the partition function for this ion using this expanded set. At 6000 $\mathrm{K}$, the new partition function, based on 407 levels, is only $1.8 \%$ larger than that found using the 354 levels included in AEL. However, at $10000 \mathrm{~K}$, the difference increases to more than $11 \%$, or about 0.05 dex. Our experience with holmium leads us to expect that the uncertainties in the theoretical energy levels (which are of the order of $100 \mathrm{~cm}^{-1}$ or less) do not compromise the accuracy of the resulting partition functions in any significant way.

W II: Ekberg, Kling and Mende (2000; hereafter EKM) have presented an analyses of the low configurations of the second spectrum of tungsten yielding 76 even parity and 187 odd parity levels. Their work adds 13 new levels below $30000 \mathrm{~cm}^{-1}$ to what is included in AEL, for an increase of about $30 \%$ over what is available from this source. At present, the levels below this threshold deriving from the $(5 \mathrm{~d}+6 \mathrm{~s})^{5}$ group are complete. We have up-dated the AEL data by including the new levels from EKM up to $40000 \mathrm{~cm}^{-1}$ and have re-evaluated the partition function for temperatures in the range 3000 to $34000 \mathrm{~K}$. At the temperature of the Sun, the differences are small (18.08 based on 137 AEL levels vs. 18.28 based on 173 levels from EKM), and increase only to about $8 \%$ at 10 $000 \mathrm{~K}$. Although the changes brought about by the availability of the new levels are modest, we urge practitioners to incorporate the EKM results into their analyses.

Re I: Wyart (1978) made a systematic study of the $(5 \mathrm{~d}+6 \mathrm{~s})^{7}$ group in Re I, discovering four new even levels, all lying between 30000 and $40000 \mathrm{~cm}^{-1}$, and re-interpreting two other levels originally described by Trees (1958). The level structure for this ion is essentially complete below about $30000 \mathrm{~cm}^{-1}$. Incorporating these small modifications into the AEL data results in essentially no change in the partition function at stellar temperatures for which this ion is likely to contribute meaningfully to the spectrum. 
Re II: AEL lists a total of 49 even parity levels for this ion; all are below 40 $000 \mathrm{~cm}^{-1}$ and most have no term assignments. Only a few contributions from the $5 \mathrm{~d}^{5} 6 \mathrm{~s}$ and $5 \mathrm{~d}^{4} 6 \mathrm{~s}^{2}$ configurations are identified, and none from the $5 \mathrm{~d}^{6}$ are reported. The odd parity levels arising from the $5 d^{5} 6 p$ configuration start at 44 $000 \mathrm{~cm}^{-1}$.

We have made preliminary calculations of the placement of the levels from the $(5 d+6 s)^{6}$ group for this ion, and find that as many as 35 (out of a total of 142) should reside below about $30000 \mathrm{cn}^{-1}$. This comports well with the 33 even levels given in AEL with energies below this threshold. Thus, we expect that the level structure for this ion is essentially complete below $30000 \mathrm{~cm}^{-1}$ and that the partition function derived from the AEL data should be reliable. We have not, however, recomputed the partition function for this ion using any portion of the high resolution experimental FTS data described by Wahlgren et al. (1997), nor have we tried to compare our Cowan code calculations with the more detailed ones of Henderson et al. (1999).

Os I: Energy levels belonging to the $(5 d+6 s)^{8}$ group for this ion have been classified by Gluck et al. (1964). Forty-six even levels were interpreted, including six that are not in the AEL data; most of the new levels are between 20000 and $30000 \mathrm{~cm}^{-1}$. Thirty-two new odd levels were classified, the lowest lying four of which occur between 27000 and $40000 \mathrm{~cm}^{-1}$. This work renders the level structure for Os I essentially complete below $30000 \mathrm{~cm}^{-1}$, but, given the small number of additions to the AEL tabulation, does not substantially change the partition function derived from the AEL data.

Os II: AEL gives 22 even levels, all of which have energies below about 25 $000 \mathrm{~cm}^{-1}$. Only five of these have been assigned to the $5 \mathrm{~d}^{6} 6 \mathrm{~s}$ configuration. The 17 odd levels identified start at about $37000 \mathrm{~cm}^{-1}$, and only five are assigned to $5 d^{6} 6 p$. Van Kleef and Klinkenberg (1961) include only four new, high-lying odd levels not present in the AEL tabulation.

We have carried out Cowan code calculations for the $(5 \mathrm{~d}+6 \mathrm{~s})^{7}$ group to better establish the energy level structure for this ion for the purposes of refining the partition function. The calculations have been carried out in the same fashion as was done for holmium (Bord and Cowley 2002), and the computed energies were fitted to the AEL values by the method of least squares. The average deviation between the calculated and experimental energies for the 22 known levels was $72 \mathrm{~cm}^{-1}(0.3 \%)$ over a range of $25000 \mathrm{~cm}^{-1}$ with the best fit atomic parameters.

Using the AEL data, supplemented only by the van Kleef and Klinkenberg results, the 43 known energy levels yield a partition function at $10000 \mathrm{~K}$ of 33.46. At the same temperature, these data augmented by our calculations (for a total of 140 levels) give a partition function of 44.89 . This is a $34 \%$ increase in the partition function. At the temperature of the Sun $(\approx 6000 \mathrm{~K})$, the new partition function is only $12.5 \%$ larger. Thus, the addition of the new calculated levels will not alter the solar abundance of osmium by more than 0.05 dex, but it could affect abundance determinations in hotter stars by nearly 0.13 dex.

\subsection{The Influence of Levels Above Those Tabulated}

It is well known that the partition function for a single atom, isolated in the universe, would diverge if the temperature were finite. It is also known that 
this odd situation is irrelevant in the real world, because the sizes of atoms are limited by perturbations from nearby atoms and ions. These perturbations lower the ionization energy and terminate the partition function. Simplistic treatments of the high levels make use of a hydrogenic approximation, and add levels until some cut-off energy is reached. This cut-off is typically equal to the first ionization limit ("the" ionization energies of Table 1) reduced by some amount. Often, only perturbations due to free charges are considered, in which case, the energy reduction is $\alpha Z e^{2} / \rho_{D}$, where $Z=1$ for a neutral atom, etc., $\rho_{D}$ is the Debye length, and $\alpha$ is a dimensionless constant of order unity.

If the degree of ionization is low, as in the case of cool stars, ridiculously inflated partition functions may result if only Debye fields are considered. If the free electron density is many orders of magnitude lower than the gas pressure, atoms will be ionized by interactions with neutral particles, in which case, the ionization energy is lowered by an amount about equal to $\beta e^{2} / \bar{r}$, with $\bar{r}$ the mean distance between atoms, and $\beta$ another constant of order unity.

Halenka (cf. http://draco.uni.opole.pl/ halenka/; Halenka and Madej 2002) and his coworkers have considered multiple ionization limits that occur in complex atoms. We refer to their papers, and the book by Griem (1997) for details.

All of these complications may be irrelevant in most cases of interest to those interested primarily in calculating line strengths in stellar spectra. The reason for this fortunate circumstance was pointed out long ago by Van't Veer-Menneret (1961): At temperatures where these high levels would become occupied, the atom or ion is mostly ionized to the next stage, and the bloated partition function will cancel from the combined Boltzmann-Saha relations which give the number of atoms capable of absorption. This provides the basis for focusing on levels below $40,000 \mathrm{~cm}-1(4.95 \mathrm{eV})$ when computing partition functions for most atoms/ions of importance in cooler stars.

To our knowledge, this comforting situation has not been studied since Van't Veer-Menneret's work, and probably should be tested in various relevant portions of parameter space.

\section{Sources of Atomic Data}

The most convenient sources of atomic data for spectral synthesis are the Kurucz CDRoms and his website (http://cfaku5.harvard.edu), and VALD Kupka, et al. 1999, see http://www.astro.univie.ac.at/ vald/). A number of more general data bases are available, many linked to one another. Two examples are:

- http://urania.astro.spbu.ru/staff/afk/AtDatCentre/DtBases/db.html

- http://plasma-gate.weizmann.ac.il/DBfAPP.html.

For individual features (lines, elements), literature searches are facilitated by on-line resources such as the Web of Science and the preprint file on astro-ph:

-http://isiknowledge.com/

-http://xxx.lanl.gov/archive/astro-ph.

The search engines of common browsers (e.g. google.com) often produce useful material.

We will comment below on some of the url's which are collected for the reader's convenience. They lead to codes, and additional references to atomic data. These lists are in no way complete, and there is considerable overlap. As 
hardware and organizations change, url's become invalid, and the irritating "url not found" may appear. Often, the relevant material is still available on line but with a different or slightly modified url. Additional surfing efforts may be rewarding.

- http://physics.nist.gov/PhysRefData/contents.html

- http://physics.nist.gov/PhysRefData/Handbook/index.html

- http://ccp7.dur.ac.uk/library.html

- http://cdsweb.u-strasbg.fr/topbase.html

- http://www.astro.lu.se/ hampus/astrophys.html/

- http://tlusty.gsfc.nasa.gov/

- http://www.umh.ac.be/ astro/dream.shtml

- http://verdi.as.utexas.edu/

- http://uw.physics.wis.edu/ lawler/

- http://star.arm.ac.uk/ csj/Welcome.html

- ftp://cdsarc.u-strasbg/cats/J/A+A (or other journals)

- http://hebe.as.utexas.edu/at/at.cgi

Generally speaking, data from the Kurucz or VALD sites will provide the basis for an encouraging synthesis of atomic features in most stars. Details are another matter. Even within small wavelength intervals, of 5 to $10 \mathrm{~A}$, there are usually a few features for which one cannot find an appropriate atomic line, even by modifying the oscillator strength by an order of magnitude or more. This is typical in a region with molecular features, when using VALD, which had not (as of May 2002) yet included molecules. Steps to change this are in the works.

Anomalously strong or missing lines can also arise from shortcomings of atomic data: Either the lines are not in the data bases, or the oscillator strengths are egregiously in error. We must recognize the difficulty of the task of providing useful oscillator strengths for all transitions of possible use in complex spectra such as Fe I. Figure 1 shows a comparison of $\log (g f)$ taken from the Kurucz and VALD sites in the spring of 2002 for wavelengths in the region $\lambda \lambda 4000-4390$. The VALD values are essentially those from Kurucz (1994), while the K99 values were taken from the Kurucz website (cfaku5, see above) in May of 2002. While the majority of the lines are from theoretical calculations, both lists contain gfvalues from NIST compilations (e.g. Fuhr, Martin, and Wiese 1988). These lines would agree perfectly, or very closely, depending on the epoch of the evaluations. All lines arise from classified (accurately known) levels.

The community owes an enormous debt to the compilers and creators of these data bases. The number of people actively working on VALD is very small, while Dr. Kurucz is personally responsible for much (if not most!) of the data available in his CDRoms and website. The Kurucz site include standard line-broadening parameters for van der Waals, Stark, and natural broadening. Information for the effects of the atomic nucleus (hyperfine structure, isotope shifts) are also available at his site. The newest file formats will have fields for this information for each wavelength, but at present, most fields have only "placeholder" entries. VALD also lists standard line broadening parameters for each line where available. 
Comparison of $\log (\mathrm{g} f)$ 's in VALD and Kurucz

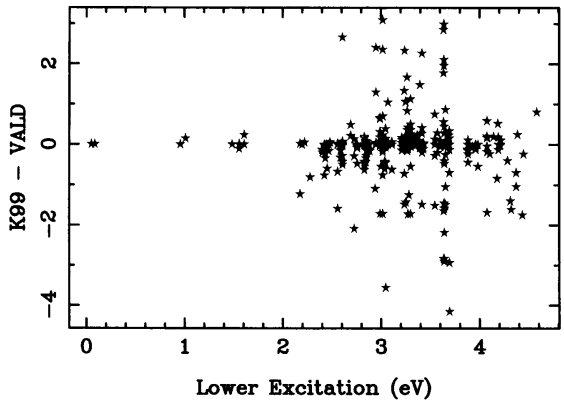

Comparison of Log(gf)'s in VALD and Kurucz

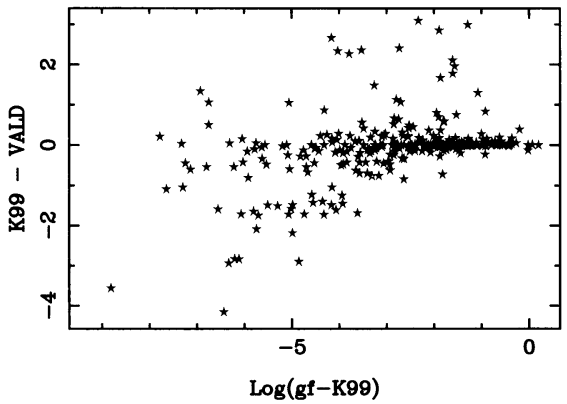

Figure 1. Comparisons of Fe I $\log (g f)$ values in from VALD and the Kurucz site as of May 2002. See text.

It is inevitable that there will be time lags between the availability of new material and its incorporation in the data bases. Having made this clear, it is only prudent to be aware of the shortcomings of this material, both from the point of view of future improvements and making wise use of the data currently available. At present, we favor the Kurucz site for iron and iron-group spectra, and VALD for heaver elements.

It is often helpful to consult recent publications or preprints to obtain atomic data. The url's given at the beginning of this section are useful starting points. Even when data is not given specifically, one may consult references and download PostScript ${ }^{1}$ versions. Tables may be converted to ascii text using software such as Ghostscript and Ghostview or similar packages. Many tables in published journals are available in ascii via ftp from the Data Center in Strasbourg (see ftp site above).

\section{Photoionization Cross Sections: Implementation of TopBase}

While detailed photoionization cross sections have been available from The Opacity Project (cf. Seaton 1987) for more than a decade, the original form was not readily adaptable to synthesis codes. TopBase data files generally consist of several hundred energy levels, corresponding to spectral terms, but without the fine-structure splitting. For example, the ${ }^{2} \mathrm{P}_{1 / 2}$ and ${ }^{2} \mathrm{P}_{3 / 2}$ of the upper terms of the $\mathrm{Na}$ I D-lines are represented by a single energy. Each level may have several hundred to a thousand photon energies, some very closely spaced in order to reproduce "resonances" arising from levels above the first ionization energy. Pronounced lower-frequency structure may arise from such levels that strongly interact with the continuum, photoexcitation of core electrons (PEC), or Cooper minima. Minima also arise because of levels above ionization that interact with the continuum, but which are not connected to the lower level by permitted transitions (cf. Cowan 1981, Fig. 18-6).

\footnotetext{
${ }^{1}$ PostScript is a trademark of Adobe Systems Incorporated
} 

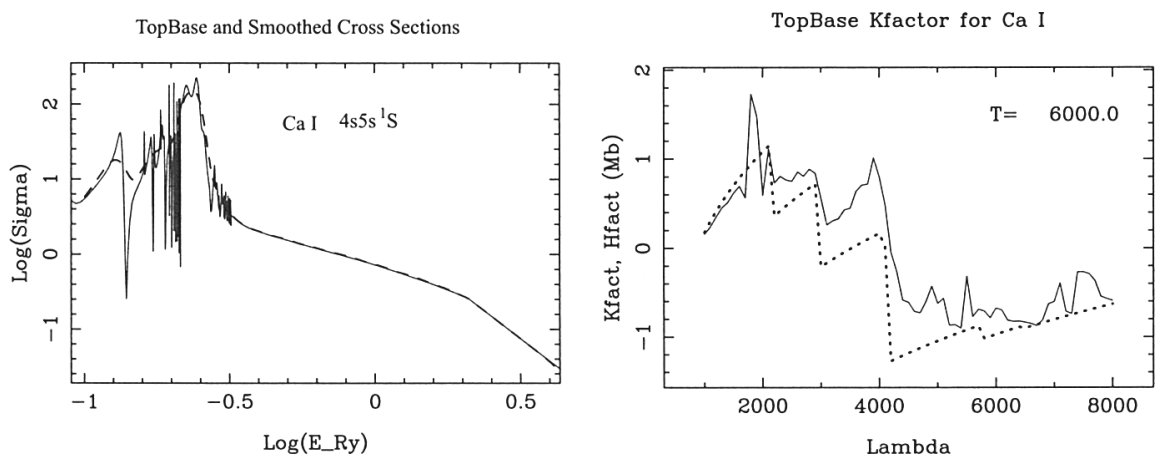

Figure 2. Left: Logarithms of photoionization cross sections vs. energy in Rydbergs TopBase. Fourier smoothed values are shown by the thick dashed line. Right: "K-factors,", or $\Sigma g_{n} \cdot \exp \left(-\chi_{n} / k T\right) \cdot \sigma_{\nu}$ vs. wavelength. The sum is over all terms in TopBase for Ca I. The dotted line shows the result if all levels are assumed to have hydrogenic photoionization cross sections. The cross sections in both panels are in Megabarns.

In addition to the sheer complexity of this detail, there has been the practical problem that the sharp resonances in TopBase are included among the Kurucz spectral lines. Moreover, the Kurucz wavelengths are correct, while the TopBase resonances have neither the correct wavelengths nor the proper fine structure. They do have proper Fano profiles and the broad PEC structures, however.

A consensus of TopBase users has emerged, that it is appropriate to smooth over the resonances with a filter that is broader than the sharper resonances, but comparable in breadth to features like the PEC or Cooper minima. Extensive calculations of this type have been carried out (cf. Prieto 2002). An example for one level of $\mathrm{Ca} \mathrm{I}$ is shown in Fig. 2 (left). The smoothing shown was done by Fourier filtering, but the result is very similar to that obtained by Prieto. Full implementation of the smoothed TopBase cross sections in routines such as ATLAS's al1op or si2op have yet be completed. These routines give Boltzmann-weighted sums of the cross sections for all levels. Partition functions are not included. The results are shown in Fig. 2 (right) for Ca I. The thick, dashed line shows the corresponding result if hydrogenic approximations are used for each level.

It seems unlikely, however, that future opacity routines will employ algorithms in which atomic levels or terms are treated individually. A more practical approach is to calculate $K$-factors, as defined in the caption above, and to do bilinear interpolation for them in temperature and wavelength. This method is already implemented in routines based, for example, on the calculations of Peach (1970). 


\section{Molecular Data}

The best single site for spectral calculations is probably that of Kurucz, which includes new calculations for $\mathrm{TiO}$ and $\mathrm{H}_{2} \mathrm{O}$. We also recommend the following sites:

- http://www.chem.msu.su/eng/raden

- http://cfs-www.harvard.edu/HITRAN/

- http://webbook.nist.gov/chemistry/form-ser.html

- http://diref.uwaterloo.ca/admin_login.html

\section{Acknowledgements}

We owe special thanks to colleagues in the atomic physics community for help and advice, and we apologize to those not explicitly mentioned. Jean Sansonetti and William Martin for provided data prior to the posting of their Handbook on the internet. We were fortunate to have advice and suggestions from Joe Reader and Wolfgang Wiese. Sveneric Johansson and Robert Cowan have been generous with their time and advice. Lawrence Lohr a physical chemist at the University of Michigan has provided much helpful advice and insight. We Thank Robert Kurucz, Tanya Ryabchikova, Nikolai Piskunov, and Fritz Kupka for numerous helpful email exchanges.

\section{References}

Bolton, C. T. 1970, Ap. J., 161, 1187.

Bord, D. J., and Cowley, C. R. 2002, Solar Phys., in press. (astro-ph/0207585) Campbell-Miller, M. D., and Simard, B. 1996, J. Opt. Soc. Am., B, 13, 2115. Corliss, C. H. 1969, J. Res. NBS, 73A, 277.

Cowan, J. J., Sneden, C., Burles, S., Ivans, I. I., Beers, T. C., Truran, J. W., Lawler, J. E., Primas, F., Fuller, G. M., Pfeiffer, B., and Kratz, K.-L. 2002, Ap. J., in press. (astro-ph/0202429)

Cowan, R. D. 1981, The Theory of Atomic Structure and Spectra, (Berkeley: Univ. California Press).

Cowley, C. R., and Barisciano, L. P., Jr. 1994, The Observatory, 114, 308.

Daems, R., Biémont, E., and Grevesse, N. 1984, unpublished results.

Dappen, W. 2000, in Allen's Astrophysical Quantities, 4th ed., ed. A. N. Cox, Chapter 3, pp. 36-38.

Ekberg, J. O., Kling, R., and Mende, W. 2000, Physica Scripta, 61, 146. (EKM)

Fuhr, G. A., Martin, G. A., and Wiese, W. L. 1988, J. Phys. Chem. Ref. Data., Vol. 17, Suppl. 4.

Gluck, G. G., Bordarier, V., Bauche, J., and van Kleef, T. A. M. 1964, Physica, 30, 2068.

Griem, H. R. 1997, Principles of Plasma Spectroscopy, (Cambridge: University Press).

Halenka, J., and Madej, J. 2002, Acta Astron., 52, 199. 
Henderson, M., Irving, R. E., Matulioniene, R., Curtis, L. J., Ellis, D. G., Wahlgren, G. M., and Brage, T. 1999, Ap. J., 520, 805.

Irwin, A. W. 1981, Ap. J. Suppl., 45, 621.

Kupka, F., Piskunov, N. E., Ryabchikova, T. A., Stempels, H. C., and Weiss, W. W. 1999, $A \& A S, 138,119$.

Kurucz, R. L. 1994, CDRom No. 22, (Cambridge MA: Smith. Ap. Obs.) See also his web site, url above.

Laun, D. D., and Corliss, C. H. 1968, J. Res. NBS, 72A, 609.

Leckrone, D. S., Proffitt, C. R., Wahlgren, G. M., Johansson, S. G., and Brage, T. 1999, Astron. J., 117, 1454.

Martin, W. C., Zalubas, R., and Hagan, L. 1978, Atomic Energy Levels-The Rare Earth Elements, NSRDS-NBS 60 (Washington: U. S. Gov. Print. Off.).

Moore, C. E. 1958, Atomic Energy Levels, Vol. III, NBS Circ. 467 (Washington: U. S. Gov. Print. Off.). (AEL)

Moore, C. E. 1970, Ionization Potentials and Ionization Limits Derived from the Analysis of Optical Spectra, NSRDS-NBS 34, (Washington: U. S. Gov. Print. Off.).

Peach, G. 1970, Mem. R.A.S., 73, 1.

Prieto, C. A. 2000, http://hebe.as.utexas.edu/at/at.cgi

Sansonetti, J., and Martin, W. C. 2000, http://physics.nist.gov/PhysRefData/Handbook/index.html

Seaton, M. J., 1987, J. Phys. B., 20, 6363.

Shadmi, Y., and Caspi, E. 1968, J. Res. NBS, 72A, 757.

Traving, G., Baschek, B., and Holweger, H. 1966, Abh. Hamburger Sternw., 8, 3.

Trees, R. E. 1958, Phys. Rev., 112, 165.

van Kleef, T. A. M. and Klinkenberg, P. F. A. 1961, Physica, $27,83$.

Van't Veer-Menneret, C. 1961, CR, 253, 380.

Wahlgren, G. M., Johansson, S. G., Litzén, U., Gibson, N. D., Cooper, J. C., Lawler, J. E., Leckrone, D. S., and Engleman, P. 1997, Ap. J., 475, 380.

Wahlgren, G. M., Leckrone, D. S., Brage, T., Proffitt, C. R., and Johansson, S. G. 1998, in The Scientific Impact of the Goddard High Resolution Spectrograph, eds. J. C. Brandt, T. B. Ake III, and C. C. Petersen, ASP Conf. Ser. 143 (San Francisco: ASP), p. 330.

Wyart, J.-F. 1978, Physica Scripta, 18, 87. 